\title{
Low complexity DCO-FBMC visible light communication system
}

\author{
Abdullah Ali Qasim¹, M. F. L Abdullah' ${ }^{2}$, Husam Noman Mohammedali ${ }^{3}$, Rahmat Bin Talib ${ }^{4}$ \\ Mohammed N. Nemah ${ }^{5}$, Ahmed T. Hammoodi ${ }^{6}$ \\ ${ }^{1,2,4,6}$ Department Communication Engineering, University Tun Hussein Onn Malaysia, Malaysia \\ ${ }^{1,3,5}$ Engineering Technical College-Najaf, Al-Furat Al-Awsat Technical University, Iraq
}

\begin{tabular}{l} 
Article Info \\
\hline Article history: \\
Received Feb 25, 2019 \\
Revised Sep 30, 2019 \\
Accepted Oct 8, 2019 \\
\hline
\end{tabular}

\section{Keywords:}

Complexity

DCO-FBMC

Hermitian symmetry

Real signal

VLC

\begin{abstract}
Filter Bank Multicarrier (FBMC) is a new waveform candidate in the visible light communication system (VLC). FBMC is a distinctive kind of multi-carrier modulation that can be regarded as an alternative to orthogonal frequency Division Multicarrier (OFDM) with $\mathrm{CP}$ (cyclic prefix). DCOFBMC (DC-bias optical FBMC) has recently been used in VLC, because it overcomes all defects in the optical-OFDM system and has high spectral efficiency. But at the same time the traditional DCO-FBMC suffers from high complexity due to the use of Hermitian Symmetry for real signal, by using $2 \mathrm{~N}$ point subcarrier IFFT (Inverse Fast Fourier Transformer) in the modulator, and the output is N-point subcarrier FFT (Fast Fourier Transform) in the demodulator. In this paper, for the first time, the possibility of minimizing complexity and generating a real signal without the use of Hermitian Symmetry or any other technique has been verified. The proposed technology provides $50 \%$ of the size of the IFFT / FFT and this results in a significant reduction in power consumption and occupied chip area.
\end{abstract}

Copyright $(2020$ Institute of Advanced Engineering and Science. All rights reserved.

\section{Corresponding Author:}

Abdullah Ali Qasim

Department Communication Engineering,

University Tun Hussein Onn Malaysia,

Parit raja, 86400 Batu pahat, Johor, Malaysia.

Email: alzubydea@gmail.com, alzubydea@atu.edu.iq

\section{INTRODUCTION}

VLC has attracted high interest from researchers because of its unlicensed bandwidth, the fact that these systems use visible light that has no electronic interference or health risk. Intensity modulation at the transmitter and direct detection at receiver (IM/DD) is customarily applied in VLC systems, because of its simplicity [1]. Therefore using the technique mentioned above, this confirms that the signal transmitted through the light source (light emitting diode (LED) or laser diode (LD)) is in the form of optical power [2], indicating that it is a positive light signal [3]. The wavelength electromagnetic spectrum range in VLC from 400 to $700 \mathrm{~nm}$ [4].

One of the most common problems facing this VLC is the limited bandwidth modulation, so the trend is to use highly efficient modulation systems, to overcome this problem, many modulation schems were used, and the most prominent of which is the OFDM technique in VLC [5]. The transmitted signal must be real to achieve this is used Hermitian-symmetry [6,7]. OFDM schemes such as DC bias optical OFDM (DCO-OFDM), and the other types of schemes each scheme has its pros and cons $[8,9]$. Researches has found that OFDM suffers from limited bandwidth due to the presence of CP OFDM suffers from leakage spectrum because of its large side lobes [5]. As in RF communication system, FBMC was considered a strong competitor to OFDM, in the fact, FBMC is a candidate waveform for $5 \mathrm{G}[10,11]$. 
According to Fourier transforms properties, for traditional OFDM, if the discrete input sequence of the modulator has its first and center coefficients null, and if it presents Hermitian symmetry with respect to its center, then the OFDM time modulated signal is real [12], while existing an OFDM instituted modulation, FBMC inherits from many features related to traditional OFDM systems [13]. Nevertheless, the Hermitian symmetry of the frequency symbols ends in a duplicated size of FFT/IFFT components [14]. Consequently, to modulate $\mathrm{N}$ frequency symbols, 2N-point IFFT/FFT transforms are required [15]. This increases the complexity of the system described. In [16] and [17] the authors presented a new technique to solve this problem in the OFDM system and obtain a real signal without resorting to the use of Hermitian symmetry.

In this paper, first time investigated the new DCO-FBMC system in the VLC system without using Hermitian symmetry and get real signal compatible with IM/DD to reduce the complexity of the DCO-FBMC system and less power consuming. Moreover, the performance of the new technology has been demonstrated to have a bit error rate (BER) where it is found to do the same performance as conventional technology.

\section{CONVENTIONAL DCO-FBMC IN VLC}

FBMC is one of the most promising modulation techniques in the field of optical communications, because it provides high spectral efficiency besides other advantages. Like any modulate methods of optical communication technology, the outgoing signal must be real and positive [18]. In this kind of systems serial data are input to the division of data in parallel and are set in frequency of which usually employ offset quadrature amplitude modulation (OQAM). In order to obtain real FBMC signal the input frequency symbols to the IFFT are forced to have Hermitian symmetry [10]. The FBMC transmitted signal can be expressed as follows:

$$
x(n)=\sum_{n=-\infty}^{+\infty} \sum_{m=0}^{2 N-1} a_{m, n} g_{m, n}(t)
$$

where, $\mathrm{m}$ is the time index; and $\mathrm{n}$ is the subcarrier index; $a_{m, n}$ is the symbol (massage) being transmitted; $\mathrm{N}$ is the number of subcarriers; and $\mathrm{g}(\mathrm{t})$ is the synthesis function which maps $a_{m, n}$ into the signal space; $\mathrm{g}_{\mathrm{m}, \mathrm{n}}(\mathrm{t})$ is the shifted version in time and frequency as (2) [19].

$$
g_{m, n}(t)=h\left(n-m \mathcal{T}_{0}\right) e^{j 2 \pi / N k\left(n-\frac{L_{p}-1}{2}\right)} e^{j \Phi_{m, n}}
$$

where, $\tau_{0}$ is the symbol spacing in time; $L p$ is the length of the pulse shaping filter $\left(L_{P}=K^{*} N\right) ; K$ is the overlapping factor; $h(n)$ is the pulse shaping filter (known prototype filter). $k$ is the subcarrier index.

$$
h(n)=1+2 \sum_{k=1}^{K-1}(-1)^{k} H_{k} \cos \left(2 \pi \frac{k n}{L_{P}}\right)
$$

$H_{k}$ is set of coefficients related to $K$ [20].

As a result of the use of Hermitian symmetric and the properties of the Inverse Fast Fourier Transformer (IFFT), the imaginary signal is excluded and the outgoing signal is real as shown in Figure 1. Figure 1 shows that $\mathrm{M}=\mathrm{N} / 2$. This means that only half of the sub-carriers are used, while the other half is excluded as a result of the use of Hermitian symmetry. It is needed $2 \mathrm{~N}$ size symbol FFT to get $\mathrm{N}$ size. The system will suffer from high energy consumption and occupy higher chip area [21].
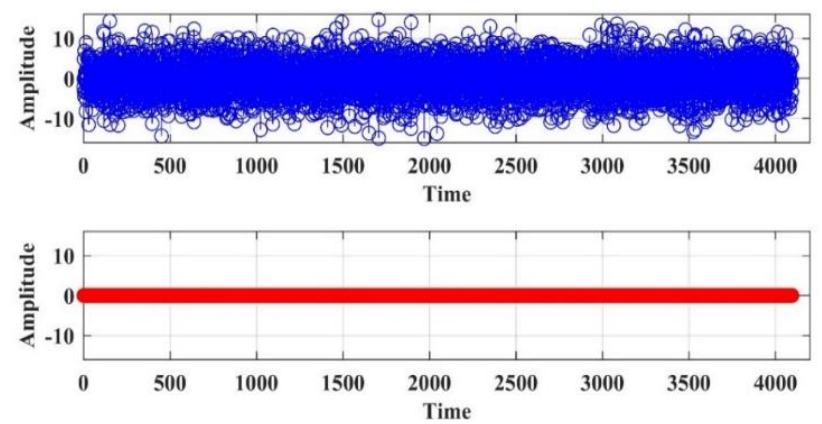

Figure 1. IFFT signal after hermitian symmetry showed the output for real signal and imaginary signal 


\section{NEW TECHNIQUE DCO-FBMC}

The use of Hermitian symmetry in modulation systems was necessary to generate a real signal, because this is a major condition in VLC, but there are disadvantages of this technique including increasing the complexity, which leads to doubling the size of IFFT/ FFT. This motivation prompted researchers in $[8,9]$ to find a new technique for generating a real signal in the OFDM system without using Hermitian symmetry. In this research, the same technique was implemented in the FBMC technique, but in different steps as follows.

\subsection{Design new optical DCO-FBMC transmitter}

The transmitted design of FBMC as shown in Figure 2, the incoming symbols are processed into the OQAM block where each symbol is filtered using the pulse shaping filter in (2) and each sub-carrier real symbol $(\mathrm{am}, \mathrm{n})$ which corresponds to the real part or the imaginary part of the complex FBMC. The transmitted signal must be a real signal, but the signal coming out of the IFFT and passing from P/S (parallel to serial converter) is a complex one (consisting of a real part and an imaginary part), and hence the imaginary part must be converted into a real part. The complex signal must be separated into two parts; real and imaginary part, then Juxtaposed technique is done which means laying the imaginary part in sequence with the real part.

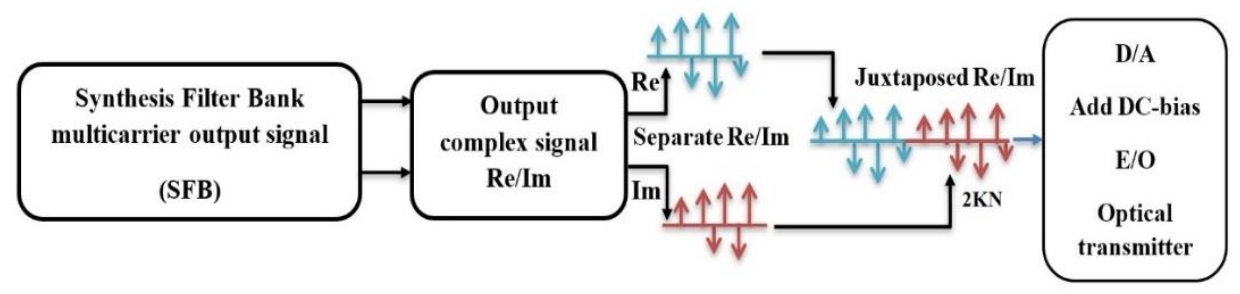

Figure 2. New DCO-FBMC optical transmitter block diagram

The frequency vector, am,n, is directly inputted to an N-point IFFT as in traditional complex FBMC systems since no Hermitian symmetry limitation is decreed, the IFFT output signal is complex and can be expressed in (4).

$$
x(n)=\sum_{n=-\infty}^{+\infty} \sum_{m=0}^{N-1} a_{m, n} g_{m, n}(t)
$$

The complex time signal can be expressed precisely in (5). This technique is applied after P/S block.

$$
x(n)=a(n)+j b(n), \quad n=1,2,3, \ldots . K *(N-1)
$$

where $\boldsymbol{a}$ and $\boldsymbol{b}$ are respectively the real and imaginary components of $\boldsymbol{x}(\boldsymbol{n})$. The KN-point real and imaginary parts of the generated in the time domain complex signal are Juxtaposed after P/S, this process generated $2-\mathrm{KN}$ real FBMC in (6).

$$
x_{2 K N}=\left\{\begin{array}{c}
a(n) \quad n=0, \ldots \ldots, K(N-1) \\
b(n-K N) \quad n=K N, \ldots \ldots, 2 K(N-1)
\end{array}\right.
$$

Figure 3 shows the real signal and the imaginary signal after their separation before applying the new technique. After that, the imaginary juxtaposed real signal in the new technique is applied as in Figure 4, thus, it is noted that the signal length is $2 \mathrm{KN}$. The signal's emerging after the application of juxtaposed technology is bipolar, for one of the most important conditions of the signal must be positive, and that is a unipolar signal. DC-biased optical FBMC is one of the simplest and primitive approaches that were proposed to generate unipolar FBMC system compatible with IM/DD systems. The DC bias desired to assure non-negativity is equivalent to the absolute value of the negative greatest amplitude of the bipolar FBMC signal. the method is to employ a DC-bias Bdc proportional to the root square of electric power $(\sigma)$ as in (7) [22].

$$
B_{d c}=b \sigma
$$

where $b$ is the clipping factor and $\sigma^{2}$ is the variance of $x(n)$ defied as,

$$
\sigma^{2}=E\left\{x^{2}(n)\right\}
$$


This is defined in [15] as a bias of $10 \log _{10}\left(k^{2}+1\right) d B$.the DC-bias

$$
x_{D C}(n)=x(n)+B_{d c}
$$

Figure 5 shows the new propose DCO-FBMC at $13.6 \mathrm{~dB}$ negative clipping.
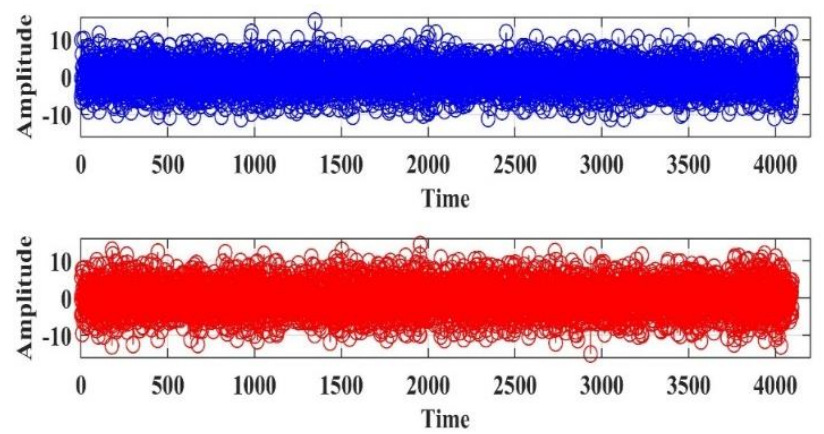

Figure 3. Output real and imaginary FBMC signal after separated

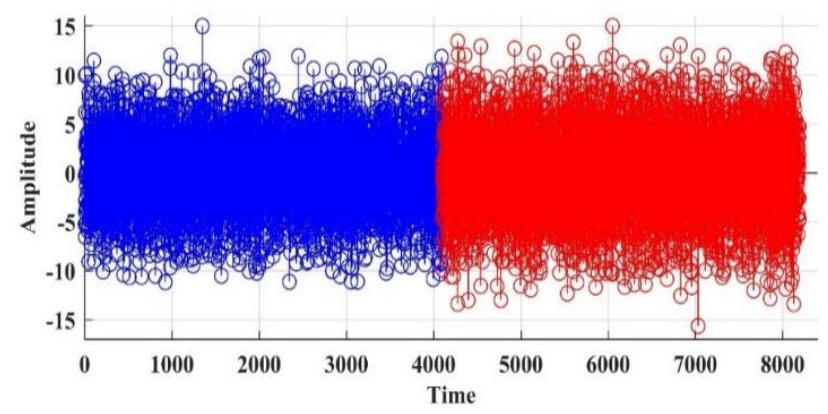

Figure 4. Imaginary signal juxtaposed to real signal at length $2 \mathrm{LP}$

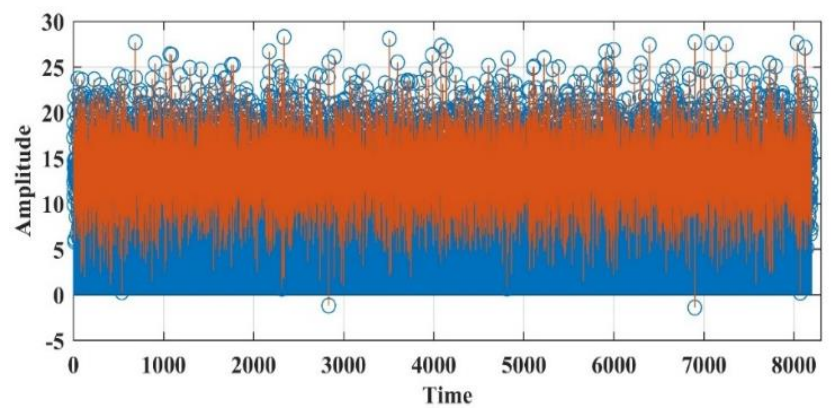

Figure 5. Add DC-bias optical to the signal at (13.6dB)

\subsection{Design new dco-FBMC receiver}

When the optical signal is received at the photodetector, the optical signal is converted to an electrical signal. Then the first step is to remove the DC-bias effect and then the real signal is separated from the imaginary part according to (10).

$$
y(n)= \begin{cases}a_{r}(n)=y_{2 K N}(n), & n=0, ., K(N-1) \\ b_{r}(n)=y_{2 K N}(K N+n) & n=0, \ldots, K(N-1)\end{cases}
$$


where $a_{r}$ and $b_{r}$ are respectively the real and imaginary components of received signal $\boldsymbol{y}(\boldsymbol{n})$ then reshaped the complex then pass the signal to analysis filter bank demodulation as the conventional FBMC system all this shows in the block diagram in Figure 6.

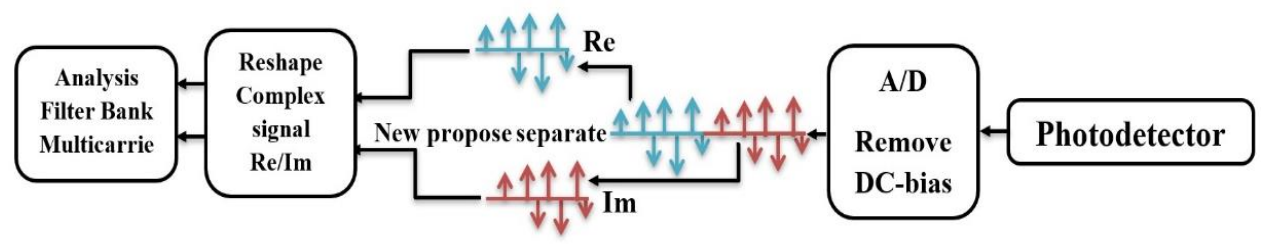

Figure 6. New design DCO-FBMC receiver block diagram

\section{COMPUTIONAL COMPLEXITY}

The FBMC technology is a complex technique, but it has advantages that distinguish it from the rest of the systems used in VLC. A new technique called Juxtaposing is proposed to reduce this complexity and obtain a real signal instead of using the traditional techniques as shown in Figure 7 and Figure 8, to calculate the complexity divided into two parts multiplications and additions using the Radix-2FFT algorithm [23]. Figure 7 and Figure 8 illustrate the comparison between the complexity of new technology and traditional technology calculated according to equation (11) and (12) for new techniques, and (13) and (14) for conventional [24, 25].

$$
\begin{aligned}
& C_{\text {mnew }}=N\left(\log _{2}(N / 2)-3\right)+8+4(N K+1) \\
& A_{\text {anew }}=3 N\left(\log _{2}(N / 2)-1\right)+8+4(N K-N+1) \\
& C_{\text {mcon }}=2 N\left(\log _{20}(N)-3\right)+8+4(2 N K+1) \\
& A_{\text {acon }}=6 N\left(\log _{2}(N)-1\right)+8+4(N K-N+1)
\end{aligned}
$$

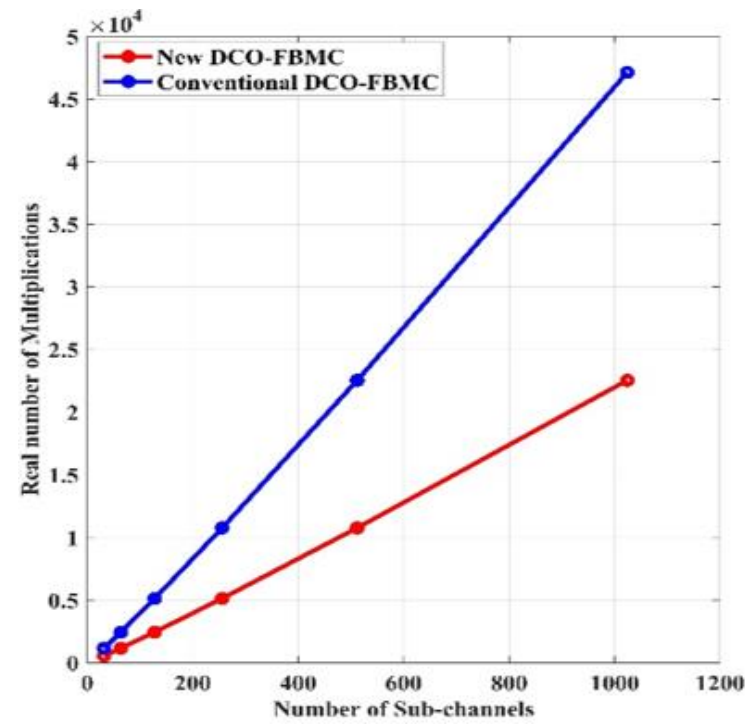

Figure 7. FBMC multiplication complexity

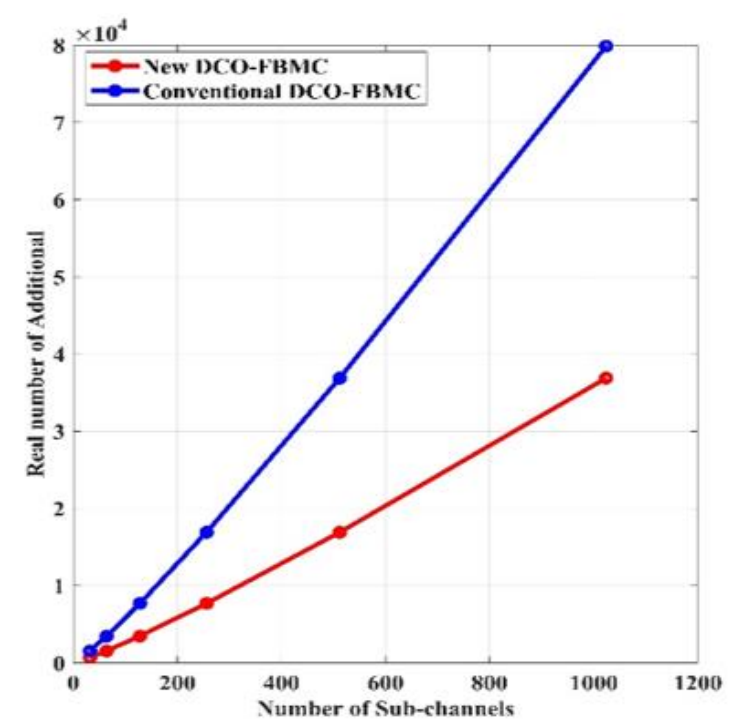

Figure 8. FBMC additional complexity

where, $C_{\text {mnew }}, A_{\text {anew }}, C_{\text {anew }}$, and $A_{\text {acon }}$ respectively are multiplications and additional calculation of new and conventional DCO-FBMC technique. The Figures explained that the new technology offers a complexity of about $50 \%$ less than conventional technology. When the FFT / IFFT size increases, both power consumption and the occupied chip area are increased. 


\section{BER PERFORMACE}

Figure 9 shows the simulation results for the BER of conventional technology and new proposed DCO-FBMC technology as a signal to noise ratio (SNR) in $\mathrm{dB}$ function. These simulators were made under DCO-FBMC of 1000 symbols for various M-QAM in AWGN constellations from 4QAM to 256QAM. Therefore when using N size for FFT / IFFT to the performance of the term SNR will provide good indications of DCO-FBMC where the results are the same as the conventional technique.

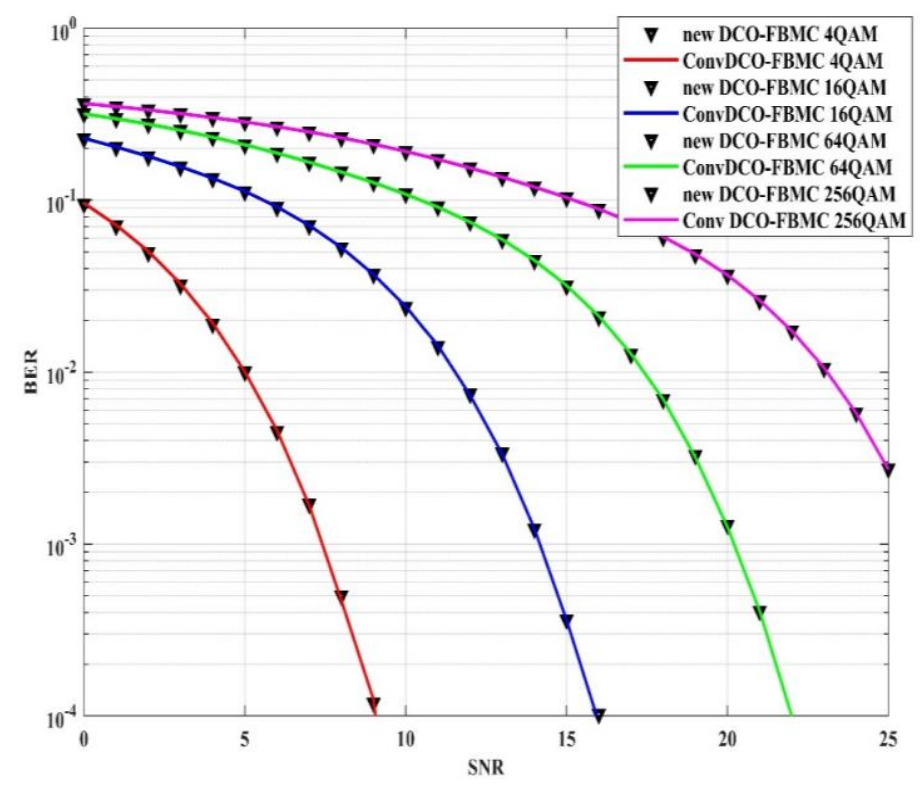

Figure 9. The BER of the function SNR dB of the New DCO-FBMC and conventional method (new DCO-FBMC IFFT size 512) and the (Conventional DCO-FBMC IFFT size 1024) for different M-QAM

\section{CONCLUSION}

In this research, new technology was created to generate a real signal from DCO-FBMC compatible with IM/DD. The proposed technique consists of generating a traditional complex FBMC signal and then extracting the real and imaginary parts juxtaposed with them in the time domain to get a real FBMC signal. This, in turn, led to a significant reduction in the complexity and thus to reduce power consumption and occupied chip area. It was also explained that the performance of the proposed FBMC technology in terms of BER is the same as the performance of traditional technology.

\section{REFERENCES}

[1] A. A. Qasim, M. F. L. Abdullah, R. Talib, H. Muwafaq, K. A. Omar, and A. M. Abdulrahman, "Visible Light Communication the next Future Generation System," 2019 Int. Conf. Inf. Sci. Commun. Technol., pp. 1-7, 2019.

[2] D. Karunatilaka, F. Zafar, V. Kalavally, and R. Parthiban, "LED based indoor visible light communications: State of the art," IEEE Commun. Surv. Tutorials, vol. 17, no. 3, pp. 1649-1678, 2015.

[3] M. S. M. Gismalla and M. F. L. Abdullah, "Optimization of Received Power and SNR for an Indoor Attocells Network in Visible Light Communication,” Journal of Communications, vol. 14, no. 1, pp. 64-69, 2019.

[4] M. F. L. A. M S M Gismalla, "Performance evaluation of optical attocells configuration in an indoor visible light communication," Indonesian Journal of Electrical Engineering and Computer Science, vol. 14, no. 2, pp. 668-676, 2019.

[5] T. Deepa, H. Mathur, N. Bharathiraja, and K. A. Sunitha, "Spectrally efficient multicarrier modulation system for visible light communication," International Journal of Electrical and Computer Engineering (IJECE), vol. 9, no. 2, pp. 1184-1190, 2019.

[6] L. Wu, Z. Zhang, J. Dang, and H. Liu, "Adaptive Modulation Schemes for Visible Light Communications Liang," J. Light. Technol., vol. 33, no. 1, pp. 117-125, 2015.

[7] A. A. Abdulkafi, M. Y. Alias, and Y. S. Hussein, "A Novel Approach for PAPR Reduction in OFDM-Based Visible Light Communications," 2017 Int. Conf. Platf. Technol. Serv. PlatCon 2017 - Proc., pp. 1-4, 2017.

[8] B. Lin, X. Tang, Z. Ghassemlooy, S. Member, X. Fang, and C. Lin, "Experimental Demonstration of OFDM / OQAM Transmission for Visible Light Communications Experimental Demonstration of OFDM / OQAM Transmission for Visible Light Communications," IEEE Photonics J. Exp., vol. 8, no. 5, 2016. 
[9] M. F. L. Abdullah, R. Green, and M. Leeson, "Optical Wireless Communication Front-Ends,” High Frequency Postgraduate Student Colloquium, pp. 3-8, 2004.

[10] R. Chen, K. Park, C. Shen, T. K. Ng, B. S. Ooi, and M. Alouini, "Visible Light Communication Using DC-biased Optical Filter Bank Multi-carrier Modulation," Glob. LIFI Congr., pp. 1-6, 2018.

[11] A. N. Ibrahim and M. F. L. Abdullah, "The potential of FBMC over OFDM for the future 5G mobile communication technology," AIP Conf. Proc., vol. 1883, 2017.

[12] J. Armstrong, “OFDM for optical communications," J. Light. Technol., vol. 27, no. 3, pp. 189-204, 2009.

[13] H. Lin, P. Siohan, C. Courtel, and C. S. Cedex, "OFDM/OQAM with Hermitian Symmetry : Design and Performance for Baseband Communication," 2008 IEEE International Conference on Communications, pp. 652-656, 2008.

[14] A. A. Abdulkafi, M. Y. Alias, and Y. S. Hussein, "Performance analysis of DCO-OFDM in VLC system," 2015 IEEE 12th Malaysia Int. Conf. Commun. MICC 2015, vol. 1, no. Micc, pp. 163-168, 2016.

[15] R. Bouziane et al., "Dependence of Optical OFDM Transceiver ASIC Complexity on FFT Size," OFC/NFOEC, vol. 1, pp. 45-47, 2012.

[16] F. Barrami et al., "A novel FFT / IFFT size efficient technique to generate real time optical OFDM signals compatible with IM / DD systems," Proc. 43rd Eur. Microw. Conf., no. 2, pp. 1247-1250, 2013.

[17] M. F. Sanya, L. Djogbe, and A. Vianou, "DC-Biased Optical OFDM for IM / DD Passive Optical Network Systems," IEEE/OSA Journal of Optical Communications and Networking, vol. 7, no. 4, pp. 205-214, 2015.

[18] J. Armstrong and B. J. C. Schmidt, "Comparison of asymmetrically clipped optical OFDM and DC-biased optical OFDM in AWGN," IEEE Commun. Lett., vol. 12, no. 5, pp. 343-345, 2008.

[19] H.A. Ali, R.M. Zaki, M.M. Tantawy, "Overlapping factor effect on the prototype filter response proposed for FBMC transmultiplexer system," ICENCO 2018 - 14th Int. Comput. Eng. Conf. Secur. Smart Soc., pp. 109-112, 2019.

[20] E. Kofidis et al., "FBMC physical layer : a primer," PHYDYAS, pp. 1-31, 2010.

[21] M. El Tabach, P. Tortelier, R. Pyndiah, and O. Bouchet, "Diffuse Infrared Personal Optical Wireless Based on Modified OFDM/OQAM," 2008 6th Int. Symp. Commun. Syst. Networks Digit. Signal Process., no. 2, pp. 161-164, 2008.

[22] S. D. Dissanayake, S. Member, J. Armstrong, and S. Member, "Comparison of ACO-OFDM , DCO-OFDM and ADO-OFDM in IM / DD Systems," J. Light. Technol., vol. 31, no. 7, pp. 1063-1072, 2013.

[23] B. J. W. Cooley and J. W. Tukey, "An Algorithm for the Machine Calculation of Complex Fourier Series," vol. 19, no. 90, pp. 297-301, 2013.

[24] L. G. Baltar, F. Schaich, M. Renfors, and J. A. Nossek, "Computational Complexity Analysis of Advanced Physical Layers based on Multicarrier Modulation," 2011 Future Network \& Mobile Summit, pp. 1-8, 2011.

[25] A. A. Husam and Z. Kollar, "Complexity Comparison of Filter Bank Multicarrier Transmitter Schemes," 2018 11th Int. Symp. Commun. Syst. Networks Digit. Signal Process. CSNDSP 2018, no. July, 2018. 